\title{
Courrier de guerre de Loeiz Herrieu à son épouse. Considérations stylistiques et style épistolaire
}

War letters from Loeiz Herrieu to his wife: stylistic considerations and epistolary style

Daniel Carré

\section{OpenEdition}

\section{Journals}

Édition électronique

URL : https://journals.openedition.org/lbl/1212

DOI : $10.4000 / \mathrm{lbl} .1212$

ISSN : 2727-9383

\section{Éditeur}

Université de Bretagne Occidentale - UBO

\section{Édition imprimée}

Date de publication : 1 juin 2015

Pagination : 329-342

ISBN : 979-10-92331-16-5

ISSN : 1270-2412

\section{Référence électronique}

Daniel Carré, «Courrier de guerre de Loeiz Herrieu à son épouse. Considérations stylistiques et style épistolaire », La Bretagne Linguistique [En ligne], 19 | 2015, mis en ligne le 01 mai 2021, consulté le 22 mai 2021. URL : http://journals.openedition.org/lbl/1212 ; DOI : https://doi.org/10.4000/lbl.1212

La Bretagne Linguistique est mise à disposition selon les termes de la Licence Creative Commons Attribution 4.0 International. 
Daniel CARRÉ*

\section{Courrier de guerre de Loeiz Herrieu à son épouse. Considérations stylistiques et style épistolaire}

\section{Pour situer la question}

$\mathrm{E}_{\mathrm{n}}$

ntre le 27 août 1914 et le 29 janvier 1919, on peut raisonnablement estimer que ce sont au moins 1100 correspondances que Loeiz Herrieu a adressées depuis le régiment à son épouse, Loeiza Ar Meliner. Un tiers peut être considéré aujourd'hui comme irrémédiablement perdu, mais les 630 courriers retrouvés et répertoriés à ce jour constituent cependant la plus importante correspondance en breton $95 \%$ du corpus est rédigé dans cette langue - pour la Grande Guerre.

La lettre, la carte est un lien, un signe que s'échangent les deux époux quasi quotidiennement. Hélas, le courrier de Loeiza n'a pas été conservé : Loeiz, comme beaucoup de soldats, l'a brûlé au fur et à mesure qu'il y a répondu.

Devant une séparation aussi longue, l'incertitude du sort de l'autre, rien d'étonnant à ce qu'on se soit beaucoup écrit entre l'avant et l'arrière. La scolarisation y avait aussi préparé toute une génération. Mais, le volume et la régularité de cette correspondanceci - pratiquement un envoi tous les deux jours - présentent un réel

* Professeur certifié breton/histoire-géographie (e.r.); Dr de l’Université de Rennes Haute-Bretagne. 
caractère exceptionnel qu'on peut expliquer par la conjonction de plusieurs facteurs :

- Loeiz écrit comme il respire; sa plume court toute seule sur le papier, sans que cela représente le moindre effort. Écrire est sa principale occupation, avant comme pendant la guerre.

- «Derhel liam er gomz » [Maintenir le lien de la parole]. C'est là, en effet, une nécessité pour les deux correspondants ; cela au-delà du lien ordinaire, naturel entre les époux. Loeiza est d'un tempérament qui la porte aisément à se faire du souci, à déprimer ; ce que Loeiz veut à tout prix éviter de crainte que cette langueur ne vienne à mettre son équilibre à lui en péril, ne risque de porter atteinte à son moral. Il faut donc maintenir un lien étroit pour répondre rapidement aux demandes, détourner le sujet s'il est considéré comme dangereux. Que cela soit fait consciemment ou non, le fait est là.

- Montrer qu'il est vivant en donnant des nouvelles du temps, de sa santé, du lieu où il se trouve (pouvoir situer quelqu'un est le savoir en vie), de ses conditions de vie (logement, nourriture), de son travail.

- Maintenir le lien avec ses parents - Loeiza leur fait part du courrier reçu, transmet leurs demandes : Loeiz ne leur adresse jamais de courrier - et les amis (transmettre les nouvelles reçues, citer les gens rencontrés au hasard des déplacements), la famille...

- Faire venir ce dont il manque au front (du beurre, des livres, quelques vêtements) et annoncer l'expédition de colis (vêtements, chaussures, tabac, objet bricolés, livres et revues...) et de mandats vers la maison.

- Répondre aux nombreuses questions de Loeiza sur la manière dont il convient d'élever leurs deux garçons, la conduite des affaires de la ferme (les abeilles, le cidre, le cheval, la voiture, les champs, l'emploi éventuel de journaliers et de servantes...), la vente des publications Dihunamb, la gestion de leur petit portefeuille de titres, les œuvres que Loeiza compose...

- Livrer à Loeiza ses pensées intimes ("Diskargein me halon" [soulager mon cœur]). Loeiz n'est en effet pas suffisamment proche de ceux qu'il côtoie - si on exclut son confesseur; mais un confesseur n'est pas un confident. Sa seule amie et confidente - il le dit lui-même - est son épouse. C'est la raison pour laquelle le personnage de Loeiz Herrieu soldat ne peut être cerné qu'au travers 
de son courrier. Kammdro an Ankoù ${ }^{1}$ ne dit en effet rien de ses pensées intimes, rien de son amour pour sa femme et ses enfants, de son respect affectueux pour ses parents ; rien encore - ou si peu - de cette forteresse intérieure dans laquelle il s'est réfugié depuis début 1916 au moins.

Il faut bien garder à l'esprit que nous nous trouvons ici au cœur d'une correspondance entre époux, un échange destiné à demeurer totalement privé et qui le serait probablement resté si le hasard de nos conversations avec Guenél Henrio ne nous avait un jour révélé son existence. Ce caractère privé fait du corpus restant un document d'une très grande valeur évidente ; non seulement du point de vue linguistique, non seulement en ce qu'il dévoile un Loeiz Herrieu intime, mais également par rapport à l'ensemble du courrier des mobilisés de la Grande Guerre.

\section{Plusieurs types de correspondance}

\section{Des supports différents}

Correspondance découverte sur des cartes "Correspondance militaire »

On peut considérer l'usage des cartes pré imprimées - on barre des mots pour constituer une phrase - comme tout à fait négligeable : le choix qui en a été fait à une ou deux reprises a sans doute été dicté par l'envie de surprendre Loeiza. Ce type de support n'est d'ailleurs disponible qu'en français.

Loeiz préfère donc rédiger. Si cela prend évidemment du temps, demande un confort d'écriture, il va s'adapter.

On peut aisément distinguer plusieurs types de cartes :

- des cartes très courtes (10 à 20 mots) :

«Tout va bien. Baisers à tous. Loeiz» (08.10.1915)

«Iah. Nitra a neùé. Karanté. Loeiz» (25.03.1916) [Bien portant. Rien de neuf. Avec mon amour. Loeiz]

1. Loeiz Herrieu, Kammdro an Ankoù, Brest, Al Liamm, 1994, 313 p. 
- des cartes courtes (10 à 100 mots) :

«Arriù onn é Berr-Mañné. Iah on ha kaer é ataù en amzér. Er gompagnoneh e arriùo arhoah vitin tré 2 eur ha 3 eur. Nitra a neùé. A galon genoh. Loeiz. Bet em es bet lihér a Loeiz e zo é Boulogne/Mer. » (14.10.1915)

[Me voici arrivé à Courtémont. Suis en bonne santé et il fait toujours beau. La compagnie rejoindra demain matin entre 2 et 3 heures. Rien de neuf. De tout cœur avec vous. Reçu lettre de Louis qui se trouve à Boulogne/Mer.]

- des cartes plus longues (100 à 200 mots)

"Me Vedig mé, arriù é hou lihér ag en 2 genein. Bremen é ha mat ha fonapl er lihérieu. Arhoah de 7 eur é han kuit. Er gompagnoneh e iei kuit de 1 eur arlerh kreisté. Heneoah é onn kouviet de goéniein get er voéz e kouskan én hé zi. Monet e hrein eit gobér plijadur dehi ha d'hé bugalé. Konz e hrér é vemb distaget ahoel épad un herrad doh er Holoniale. Keméret hon es ataù ur secteur neùe hoah. Larein e hrér e vemb dispartiet a gompagnoneheu ha lakeit de labourat ar en henteu, er linenneu, ér garieu. Mat e vehè e fehè guir en dra-sé. Aben a pe houiein un dra benak é kasein doéré d'oh. Kenevo, me Vedig a garanté. Kant bok d'oh ha d'er vugalé ha d'em zud. Loeiz. » (06.01.1916)

[Ma Fauvette chérie. Ta lettre du 2 m'est parvenue. Maintenant le courrier marche vite et bien. Je quitte ici demain à 7 heures. La compagnie partira à 1 heure de l'après-midi. Ce soir, je suis invité à dîner par la femme chez laquelle je couche. J'ai accepté l'invitation pour leur faire plaisir, à elle et aux enfants. Le bruit court que nous serions enfin détachés de la Coloniale. On vient pourtant de prendre encore un nouveau secteur. On dit que les compagnies deviendraient autonomes, qu'elles seraient affectées à la réparation des chemins et des lignes ou employées dans les gares. Tant mieux si ça pouvait être vrai. Dès que j'en saurai davantage je te le ferai savoir. Au revoir, ma Fauvette adorée. Cent baisers à toi, aux enfants et à mes parents.]

Bien sûr, l'expédition de cartes découvertes dans une langue autre que le français peut en intriguer plus d'un aujourd'hui : comment la censure pouvait-elle s'exercer? Il serait trop long de répondre ici en détail. Disons simplement que le poste occupé par Loeiz (sergentfourrier de la $3^{\mathrm{e}}$ compagnie du $1^{\mathrm{er}}$ bataillon du $88^{\mathrm{e}} \mathrm{RIT}$ ) le met, en 
temps ordinaire, à l'abri de la curiosité et des ciseaux d'Anastasie. Lorsqu'il sort de son secteur postal (pour ses missions de fourrier) ou bien encore lorsqu'il se trouve engagé dans la phase de préparation immédiate, voire de déclenchement, d'une offensive, le courrier découvert sera rédigé en français ; du moins l'accroche...

«Me V. K. - Suis en bonne santé et én hevelep léh [au même endroit]. Tout va bien. Embrasse pour Tetad [Papa] nos deux gars et les grands parents. Viens de rencontrer l'abbé Le Bihan de Languidic avec qui j'ai longuement causé. A galon [De tout cœur]. Loeiz Herrieu. » (05.05.1917)

« Sommes toujours en route! Marchant tous les jours. Santé excellente malgré fatigue.

Vous envoie bons baisers à distribuer entre vous tous plus pakad bras butum [gros paquet de tabac] et bouist amonenn [boîte à beurre] qui arriveront ces jours-ci chez M. Bayon. Loeiz Hui [Ton Loeiz]. » (25.07.1917)

\section{Correspondance cachetée (sous enveloppe)}

On peut distinguer plusieurs types de support :

- des cartes « Correspondance militaire » qui sont alors écrites recto-verso ;

- des cartes postales; au début de la guerre surtout (Reims, Fismes...). Le fourrier a facilement le loisir d'en acheter ; il s'en fait également venir de la maison. Loeiz n'utilise pas, par choix délibéré, les cartes illustrées qui ont beaucoup fleuri durant la guerre (Le baiser du poilu, La permission, etc.) ;

- des cartes-lettres militaires (les feuilles se replient ; bords autocollants) ;

- du papier à lettre ordinaire ;

- des supports « inattendus » : le verso de feuilles imprimées, du papier à en-tête (Dihunamb, Banque Populaire...), du papier ayant enveloppé un paquet de cartouches, une carte de correspondance allemande...

Il arrive souvent que le courrier commence sur un type de support pour se terminer sur un autre !

La longueur du courrier varie en fonction de plusieurs paramètres que Loeiz mentionne très souvent en introduction ou en conclusion : 
- le temps dont l'homme dispose : beaucoup de travail, passage imminent du vaguemestre, temps libre avant le repas, en attendant que la chandelle se meure...

- les conditions de confort matériel dans lesquelles il écrit : sur son genou dans un trou individuel, au milieu des conversations du « bureau », seul au milieu de la nature,...

- le nombre de lettres de Loeiza auxquelles il n'a pas encore répondu : la correspondance entre les deux fonctionne comme un dialogue, un échange de questions-réponses ;

- le nombre de jours écoulés depuis la « vraie » lettre précédente (les cartes découvertes ne sont pas vraiment des lettres).

\section{Considérations stylistiques}

Rien de nouveau dans la manière de rédiger le courrier : Loeiz suit les règles générales auquel obéit le style épistolaire français.

L'adresse : tous les courriers sont adressés à « Madame Henrio, Le Cosquer, Lanester (Mhan) ». Une carte ou deux seront cependant adressées aux enfants.

La date - y compris le jour de la semaine et parfois même le moment de la journée - est toujours mentionnée. C'est une nécessité pour le correspondant qui risque de recevoir les lettres dans le désordre et de ne plus s'y retrouver! Ceci peut déstabiliser, conduire à développer des scénarios, à entraîner des quiproquos. Loeiza, qui préfère numéroter ses courriers, se fait rappeler plusieurs fois à l'ordre : on ne sait pas quel jour elle a écrit! Loeiz n'a pas toujours un calendrier sous la main; dans la guerre, le monde de l'avant fonctionne sur un autre rythme que celui de l'arrière.

\section{L'accroche}

Me haranté,... [Mon amour] / Me haranté guellan,... [Mon meilleur amour] / Me haranté mé,... [Mon amour à moi]

Me Vedig,... [Ma Fauvette] / Me Vedig mé,... [Ma Fauvette adorée] / Me Vedig a garanté,... [Ma Fauvette que j'aime] / Me V. m. K.,... [Ma Fauvette à moi que j'aime].

Vedig an Evel [la Fauvette de l'Evel] était déjà le nom de plume de Loeiza avant son mariage. 


\section{L'entrée en matière est de plusieurs types}

- Classique :

« Un tammig amzér e chom genein... » [J'ai un peu de temps devant moi...] / «Eh on ur hard-eur dein hag e skriùan d'oh... » [J'ai un quart d'heure à moi et je t'écris...].

- Une excuse pour la brièveté, le décousu possible du courrier à venir :

«É han d'asé kas ur gir benak d'oh deusto d'en ofiserion e zo é konz ama tro ha tro d'ein » [Je vais essayer de t'écrire quelque chose en dépit du bruit que font les officiers qui sont ici à causer tout autour de moi].

- Le moment où il écrit :

«... un ériad benak de reskond d'hou lihérieu ha de gonz genoh » [une petite heure pour répondre à tes lettres et causer un peu avec toi] / « Epad men des 4 aval doar é poahein ar en tan e skriùan er pennad lihér-men d'oh » [Pendant que mes quatre patates cuisent sur le feu je t'écris cette lettre].

- L'endroit où il écrit :

« Astennet ar er plouz étal pedér buoh vras e zalh mat d'ein hag e chervij de duemmet d'em hambr (!) e skriùan d'oh» [Allongé sur la paille à côté de quatre grandes vaches qui, en me tenant compagnie, me chauffent aussi ma chambre (!), je t'écris] I «Ag en trein e skriùan d'oh, ag ur bagon loñned léh ma omb a houdé hantér-noz déh » [C'est du train que je t'écris, d'un wagon à bestiaux où nous sommes depuis hier minuit] / « $A$ me lonch, én tranché, e skriùan d'oh épad mé ma en héaul é parein ar er bratel emgann " [De mon trou, dans la tranchée, je t'écris alors que le soleil brille sur le champ de bataille].

\section{Le corps de la lettre}

- Donner de ses nouvelles.

- Le temps qu'il fait est indiqué systématiquement :

« Skornet en des ha guelarzé; marsé er fang e skorno eùé ben er fin » [Il a gelé et c'est tant mieux ; peut-être la boue finira-t-elle par geler, elle aussi] / « Hiniù é ma splann en amzér. Aerlestri zo 
lan en ér ag en amzér ha hoari e hra en treu get er hanoñneu» [Aujourd'hui, le temps est dégagé. Le ciel est plein d'avions et l'artillerie s'est mise de la partie].

- La santé est abordée également à chaque fois :

«Vais bien. Suis reposé »; «Iah ha mat en treu » [Suis en bonne santé et tout va bien] / «Ne harzan ket mui get me zreid. Er hig e zo saùet tro ha tro d'em ivineu ha foèuet » [Mes pieds me font terriblement souffrir. Mes orteils ont enflé au point que la chair s'est soulevée autour des ongles].

- Quelques nouvelles de la guerre :

«Ni zo ni ataù é Saulchoy ha rezi e zo e chomemb hoah un herrad ha guelarzé " [Nous, nous sommes toujours à Saulchoy et tout laisse à penser que nous y séjournerons encore quelque temps. Tant mieux] / « Arhoah vitin é hamb de gemér hon tranchéieu da 4 eur » [Demain matin nous irons prendre nos tranchées à 4 heures] / " Hiniù é han de Fismes de brenein treu » [Aujourd'hui je vais à Fismes faire des achats] / « Déh d'en noz hor boè boutet potarneu muioh eit bihannoh ar gein er Germaned ken e vleijent! » [Hier au soir, notre artillerie a marmité les Allemands plus que jamais. Au point de les faire hurler !]

- Des nouvelles des gens connus de Loeiza :

«En E. Gal e zo fondet é zorn get un tam potarn» [Monsieur Le Gall a été blessé à la main par un éclat d'obus] / « Guélet em es Gloahec » [J'ai rencontré Gloahec].

- Les lettres, journaux, paquets reçus :

"Arriù é en amonen genein. Nen des chet ridet» [Le beurre est arrivé. Il n'a pas coulé] / «Er chokolat e zo deit d'ein én ur pakad» [Le chocolat m'est arrivé en un bloc] / « Mat é er pélerine » [La pèlerine convient parfaitement] / « Kroaz er Vretoned hag en Nouvelliste e zo deit de gas d'ein keveleu ag er vro»" [Kroaz ar Vretoned et Le Nouvelliste sont venus m'apporter des nouvelles du pays].

- Dire ce qu'il a fait porter, ce qu'il a expédié à la maison :

« 4 pakad butum em es kaset d'em zad» [J'ai expédié 4 pa- 
quets de tabac pour mon père] / « Kaset em boè hoah déh ur pakad de di Bayon » [Hier, j'ai encore fait partir un paquet qui sera remis chez Bayon] / « Kas e hran ur mandat a 50 livr d'oh» [Je t'envoie un mandat de 50F].

- Demander des nouvelles des enfants, de ses parents, des proches, des voisins... :

« Na Herp, penaus é ha geton? Laret dehon é suhetan dehon donet de blom » [Et Herpe, comment se porte-t-il ? Dis-lui que je lui souhaite un bon rétablissement].

- Demander des nouvelles de la ferme, des affaires :

«Bout e hues bouisteu pé ruchenneu prest eit kemér er gurén?»[As-tu des ruches ou des caisses pour recevoir les essaims ?] / «Koutant onn é ma saùet hou koeidi » [Je suis content d'apprendre que les oies sont bien venues] / «Penaus é ta en treu en doar (bouid loñned) ? Nag er gurén? » [Comment se présentent les cultures (les fourrages) ? Et les abeilles ?]

- Demander qu'on fasse quelque chose pour lui :

«Hui hello davé amonen d'ein arré » [Tu peux m'expédier du beurre à nouveau] / « Kaset ur pakad bouistadigeu konserv d'ein » [Expédie-moi des conserves en boîte] / " Pédet aveit en divroet » [Priez pour l'exilé] / "Kaset d'ein dré ti Bayon deu livr: Chansons Populaires du Pays de Vannes » [Fais-moi parvenir par chez Bayon deux exemplaires de Chansons Populaires du Pays de Vannes].

- Répondre aux demandes de Loeiza, donner son avis. La présentation - tiret, à la ligne en début de rubrique - indique clairement qu'il répond directement aux questions de son épouse dont il a le ou les courriers sous les yeux. De nombreux thèmes sont évidemment abordés : la durée de la guerre, l'éducation des enfants, la manière de conduire la ferme (le cidre, les abeilles, les cultures en général), les amis et voisins, se soigner, l'avenir...

« Aveit er brezél, dén ne uél hoah pegours é achiùo » [En ce qui concerne la guerre, personne ne peut encore dire quand elle prendra fin] / « Er liorh ardran en ti é hellér torrein en doar de bas er gouian, ha dihoal mat a dorein en distéran planten ag er 
ré em es laket, a ziranj er gurén » [Dans le courtil de derrière on peut bêcher avant l'hiver; il faut cependant bien faire attention à ne pas abîmer l'une ou l'autre des plantes que j'y ai installées, à ne pas déranger les abeilles] / «Asé teillat er prad er blé-men get teil ag er hreu. Ma nen des chet erhoalh lakat scories » [Essaie de fumer la prairie cette année avec le fumier de l'étable. Si c'est trop juste, mettre des scories] / «A pe vo amzér : turel ur sél ar me vélo aveit en nétat » [Quand vous aurez le temps, il faudrait jeter un coup d'œil sur mon vélo, le nettoyer] / «Enep d'er grip é ma ret um zerhel tuem mat, pas ivet nitra iein ha kemér grogeu» [Contre la grippe il faut rester bien au chaud, ne pas boire froid et prendre des grogs] / « Mad e hret diskein er vugalé en ur hobér plijadur d'ou spered : elsé ne zihousteint ket» $[\mathrm{Tu}$ as raison de faire appel au plaisir intellectuel des enfants en les instruisant : ainsi ils ne se détourneront pas de l'instruction] / " Kemér pasianted, karein er boén chetu en deu dra e faut de vab dén eit bout eurus memb ér goaleur » [Savoir se résigner, aimer souffrir voilà deux choses indispensables à l'homme qui veut être heureux en dépit du malheur].

- S'épancher, se confier, tranquilliser :

« Stert é bout dispartiet, stertoh hoah eit er ré um gar mat... » [La séparation est une dure chose, bien plus dure encore pour les gens qui s'aiment] / «Na kaeroh é éleih plijadur deu spered e blij en hevelep treu dehè, diù galon e gar en hevelep treu hag um gar mat étrézè » [Bien plus beau est encore le plaisir qu'éprouvent deux esprits appréciant les mêmes choses, deux cœurs ayant les mêmes penchants et s'aimant bien l'un l'autre] / «Liés é ta chonj d'ein ahanoh, ag en noziehadeu bourus hon es treménet ér Gohkér é divréh en eil égilé... » [Souvent, je songe à toi, aux nuits ennivrantes du Cosquer passées dans les bras l'un de l'autre] / «Ne geméret ket poén erbet genein» [Ne vous faites pas de souci pour moi] / «Bremen é toujan a huélet er vuhé goudé er brezél ken trist èl en hani e zo bet ret d'ein biùein a houdé 1914 » [Maintenant, je crains que ma vie après la guerre ne soit aussi triste que celle que j'ai dû mener depuis 1914].

\section{La conclusion}

Elle est le plus souvent assez banale, convenue :

« Arriù é eur ar pred... » [Voici l'heure du repas] / « Nozeh 
vat, me Vedig... » [Bonne nuit, ma Fauvette] / «Labour e zo dreist penn ha... » [Du travail par-dessus la tête, aussi...].

Il arrive cependant qu'elle traduise l'état physique ou moral dans lequel se trouve le rédacteur, qu'elle offre un coup d'œil furtif sur une scène pleine de vie :

«Kenevo, me haranteig. Éma Er Fol é arriù de sinein ar papérieu hag é pad ma vo ama kenevo d'em lihér » [Au revoir, mon cher Amour. J'aperçois Le Fada qui s'en vient signer les pièces du jour et, pendant qu'il sera ici, adieu ma lettre]/ «Kenevo, me Vedig mé. Ne uélan ket mui hag aneouid em es» [Au revoir, ma chère Fauvette. Je n'y vois plus goutte et j'ai froid] / «E han d'hou lezel, Me Vedig, rak goust ebet de skriù $n$ 'em es chet» [Je vais te quitter, ma Fauvette : l'envie me manque d'écrire plus longtemps] / «Kenevo, me Vedig. Lan é en ti a dud arré ha barbot! barbot! » [Au revoir, ma Fauvette. La maison est à nouveau pleine de monde et ça jacasse ! Ça jacasse !]

Avant la signature viennent souvent des appels à la protection céleste :

«Doué d'hou koarn hag er ré e garamb» [Dieu te garde ainsi que tous ceux qui nous sont chers] / "Doué d'hou koarn d'ein rah» [Dieu veuille vous conserver tous à moi] / «Doué d'hou koarn hou pempig » [Que vous garde, mes chers cinq] / «Doué ha Sant Guénel beniget d'hou koarn d'ein » [Que Dieu et StGwenael béni veuillent vous conserver à moi].

\section{Pour prendre congé}

Au-delà du «Kenevo d'oh!» [Au revoir !], diverses formules sont utilisées; le choix de l'une ou de l'autre est souvent guidé, inconsciemment sans doute, par le ton et le contenu de lettre :

«D'oh hou pemp rah me chonjeu karantéusan " [À vous, mes chers cinq, tout mon amour] / «Um garet étrézoh hou pemp é chonjal en hani hou kar drest pep tra » [Aimez-vous bien les uns les autres en pensant à celui qui vous aime par-dessus tout] / «A galon» [De tout mon cœur] / «A galon ataù » [De tout mon cœur, comme toujours] / " A galon genoh » [Avec vous de tout mon cœur] / «A galon hag a spered genoh » [Avec vous de tout mon cœur, de tout mon esprit] / «Kant bok» [Cent baisers] / 
«Beb a vok d'oh rah» [Un baiser à chacun d'entre vous] / "Karanté d'oh rah» [Je vous aime tous] / "Karanté a iuh» [Je vous aime très, très fort] / «Boket me zud » [Embrasse mes parents] / «Kant bok d'em deu huil » [Cent baisers à mes deux loustics].

Parmi tous ces baisers, certains sont, évidemment, strictement réservés à Loeiza :

«Pokoù start» [Baisers bien forts] / «Pokeu niverus» [Baisers multiples] / «Pokeu tuemm skaut » [Baisers ardents] / «Pokeu loskus » [Baisers brûlants] / « Kalon oh kalon » [Cœur à cour] / «D'oh a béh » [À toi sans partage] / «D'oh dalhabl » [À toi pour toujours] / «Karanté a végad» [Amour à pleine bouche].

Signature

«Loeiz »; «Hou Loeiz » [Ton Loeiz] / «Loeiz Herrieu»; «L. H. »; «Hou parvek» [Ton poilu] / «Loeiz Vedig»; « Pepon $H u i »[$ Ton petit câlin]...

\section{Langue et style}

Le temps imparti à l'intervention ne permettait pas d'aborder cet aspect. Nous avons simplement mentionné quelques grandes lignes que nous nous contenterons de rappeler ici.

La langue employée est ce qu'on pourrait qualifier de breton de bon aloi : simple et riche pourtant, parfaitement naturel, coulant, d'une grande légèreté. Absolument rien de factice, de forcé ; aucune rature. Nous sommes au cœur d'un dialogue, d'une conversation entre deux personnes ayant une certaine éducation dont le breton est la langue de tous les jours, de toutes les circonstances de la vie.

L'écriture, à la plume le plus souvent, est très lisible.

L'attaque est généralement directe, franche, sans considérations inutiles :

« Eit an tad goei hui e rehe mad... » [En ce qui concerne le jars, tu ferais bien de...] / «Eit me veston, prenet danné... » [Pour ce qui est du veston, achète du tissu...] / « Eit en amonen de gas d'ein... » [Pour le beurre qu'il faut m'expédier...]. 
L'interrogation est toujours directe, souvent lancée par « $N a »$ :

«Na Mari Louis guel é?» [Et Marie-Louise, va-t-elle mieux ?] / " Ha reseuet e hues me fakad? " [As-tu reçu mon paquet ?] / " Nag ér Goh-kér? » [Et au Cosquer ?] / " Pesord aveleu e zo kaset d'oh? » [Quelles pommes t'a-t-on livrées ?] / « Na ré Goulian penaus é ha getè ?» [Et les Goulian, comment vont-ils ?] / « Nag er vateh, kavet hues unan? » [Et la bonne, en as-tu trouvé une ?] / «Penaus é ma seblantet en ed ? » [Comment se présente la moisson?].

Pour formuler des demandes, des conseils, voire des injonctions, le mode impératif est évidemment utilisé :

« Dihoallet doh er chistr » [Surveille le cidre] / « Ne zoujet ket lakat tud d'hou sekour » [Ne crains pas d'embaucher de la main-d'œuvre].

Il cède cependant souvent la place à des tournures moins tranchantes :

« Hui laray din ma... » [Tu me diras si...] / « Ma karehèh kas dein... » [Si tu pouvais m'expédier...] / « Koutant e vehen de... » [Je serais bien aise de...] / « Hui e rehè mat prenein ... » [Tu ferais bien d'acheter...] / « Ne faut ket ankouat penaus... » [Il ne faut pas oublier que...].

La phrase est brève. Comme on peut s'y attendre, l'ordre sujet, verbe, attribut ou complément domine ; sans pour cela omettre la mise en valeur d'un élément quand elle est souhaitée :

«Bale n'hellomb ket kalz» [Se promener, on ne le peut guère] / «Deulinein n'hellér ket » [S'agenouiller est impossible] / «Andur e zo ret » [Souffrir, il le faut].

La coordination, la juxtaposition sont très majoritairement préférées à la subordination :

« Meit didrous on ha gellout e hran lén » [Mais je suis tranquille et je peux lire] / " Get en dra-sen e vè mil boén é tostat ha ni ihuéloh eit... » [Ajoutons à cela la difficulté de l'approcher, nous qui sommes plus haut que lui]. 


\section{Conclusion}

La correspondance de Loeiz Herrieu à son épouse durant la guerre est un véritable trésor à plusieurs titres. Elle offre un complément, une mise en perspective du témoignage public livré par l'homme dans Kammdro an Ankoù. Elle constitue un corpus intéressant pour une analyse linguistique approfondie qui permettrait très certainement de mieux cerner les facettes de cette langue de bon aloi - « ar parlant onest », comme nous croyons devoir la nommer. Elle est, sans doute, la plus volumineuse correspondance privée jamais tenue en breton - au moins jusqu'en 1920 - qui nous ait été conservée. 